\title{
Évaluation d'une stratégie d'enseignement sur la dynamique du transfert des acquis de formation en situation professionnelle
}

\author{
Impact evaluation of a teaching strategy on the transfer of training
}

\author{
Sylvie KINET ${ }^{1}$, Claire MARChAND ${ }^{2}$, Jeanne RALLIER ${ }^{1}$ et Rémi GAGNAYRE ${ }^{2}$ \\ 1 Institut de formation des cadres de santé de l'Assistance Publique-Hôpitaux de Paris, France \\ 2 Laboratoire de pédagogie de la santé, EA 3412, Université Paris 13, Bobigny, France
}

Manuscrit reçu le le 15 avril 2009 ; commentaires éditoriaux formulés aux auteurs le 14 août et le 19 octobre 2010 ; accepté pour publication le 20 novembre 2010

\begin{abstract}
Mots clés :
Cadre de santé ;

compétences

en management ;

stratégie

d'enseignement ;

transfert d'acquis

de formation

Résumé - Contexte : Le management des ressources humaines est un des piliers de la fonction cadre de santé. Cela nécessite que les instituts de formation de cadres de santé construisent un dispositif de formation favorisant le transfert des acquis de formation (TAF) dans ce domaine. Objectifs : Cette étude consiste à évaluer l'impact d'une stratégie d'enseignement sur le TAF chez des cadres nouvellement formés. Il s'agit également d'identifier les éléments individuels et environnementaux influençant ce transfert. Méthode : Une enquête par questionnaire a été réalisée auprès de 122 cadres de santé ayant suivi la formation en 2007 et en poste depuis au moins quatre mois. Résultats : 37 questionnaires ont été analysés. L'analyse des situations-problèmes décrites par les cadres de santé novices montre que les acquis de formation ont été transférés par ces derniers. Ils déclarent avoir mobilisé des ressources acquises au cours de leur formation. Parmi les éléments de la stratégie d'enseignement, la préparation et l'exploitation des stages n'ont pas été considérés, par les cadres novices, très utiles pour résoudre les problèmes décrits. La plupart des cadres novices se sont estimés compétents et confiants dans la résolution de problème. Le soutien hiérarchique, les discussions avec des collègues et la période d'intégration sont, dans cette étude, des facteurs facilitant le transfert. Conclusion : Selon cette étude, les compétences en management des ressources humaines des cadres novices sont dues à différents facteurs ayant influencé le TAF. Il est envisagé d'améliorer certains aspects de la stratégie d'enseignement en lien avec les périodes de stage, dans le but de renforcer l'intégration des savoirs théoriques et pratiques.
\end{abstract}

Keywords:

Teaching strategy; managerial competence; health manager; transfer of training
Abstract - Context: Human resource management is one of the health manager's main responsibilities. Health manager training institutes need to create strategies that encourage the transfer of training (TOT). Objectives: This study proposes to assess the impact of a teaching strategy on TOT among newly trained health managers. The aim is also to identify personal and environmental factors that impact this transfer. Method: A questionnaire was sent to 122 health managers who took training in 2007 and had been working for at least 4 months. Results: 37 questionnaires were returned. The analysis of the management issues mentioned 
by newly trained health managers shows that the training assets are being transferred. Managers say that they mobilized resources acquired during training in order to solve health resource issues. In the teaching strategy, the only elements that health managers had not felt useful were those related with onsite training preparation and debriefing. Most newly trained health managers see themselves as competent and feel confident in solving the management problems identified. Upper management support, discussions with colleagues and the onboarding period are the factors that facilitated TOT. Conclusion: According to this study, human resources management skills among health managers depend on various factors that influence the TOT. Reinforcing the training strategy is being considered with regards to onsite training in order to improve the integration of practice and theory.

\section{Introduction}

En France, la mise en place effective des nouvelles organisations hospitalières (décret $\mathrm{n}^{\circ}$ 2005-421 du 4 mai 2005 portant diverses dispositions relatives au fonctionnement des établissements publics de santé et modifiant le code de la santé publique et notamment la nouvelle organisation interne en pôles d'activités) a renforcé la fonction managériale des cadres de santé de proximité. Cela suppose pour ces personnels nouvellement formés de pouvoir mettre en adéquation l'objectif visé de qualité des soins et la mise à disposition de moyens suffisants (humains et financiers) dans une économie contrainte.

L'enjeu professionnel pour les cadres responsables d'unité de soins est «d'organiser l'activité paramédicale, animer et coordonner les moyens d'un service de soins, médico-technique ou de rééducation, en veillant à l'efficacité et la qualité des prestations » (définition extraite des fiches-métier de la fonction publique hospitalière). Les 42 semaines de formation ont pour objectif de développer les capacités et les compétences nécessaires à l'exercice de la fonction de cadre de santé en secteur d'activités hospitalières. L'ingénierie pédagogique mise en œuvre lors de la formation des cadres vise à faciliter le transfert en situation des capacités acquises par les futurs cadres lors de leur cursus de formation. En effet, veiller à ce que les futurs cadres de santé puissent construire en situation une nouvelle habileté et non appliquer uniquement une habileté acquise $^{[1]}$ constitue un des buts de la formation initiale professionnelle.

Nous utilisons dans cet article le terme de «transfert des acquis de formation» au sens où d'autres auteurs se réfèrent aux notions de transfert d'apprentissage ou de compétences ${ }^{[2-4]}$. Nous nous référons à la notion «d'applications de connaissances, de savoir-faire, de comportements appris au cours d'un programme de formation sur le terrain professionnel » ${ }^{[5]}$.

Comme nous allons le développer, le lien acquis de formation - transfert - mise en œuvre de compétence n'est pas linéaire. Il dépend de facteurs liés à la formation, à l'individu et à l'environnement de travail ${ }^{[5]}$. Par ailleurs, l'article aborde principalement le versant de l'impact de la stratégie d'enseignement (entendue comme un ensemble d'activités ou séquences planifiées par le formateur) ${ }^{[6]}$ dans l'élaboration du transfert d'acquis, sans explorer de façon approfondie l'organisation du travail dont on sait également qu'elle influence les possibilités de transfert ${ }^{[5]}$. Enfin, cette étude porte sur une période de la professionnalisation de cadres de santé correspondant à leur première prise de fonction. C'est pourquoi, pour les besoins de l'étude, nous les nommerons cadres de santé « novices ».

\section{Problématique et cadre conceptuel}

\section{Quelques facteurs influençant le transfert} des acquis de formation

Une littérature abondante recense les différents facteurs influençant le transfert des acquis de formation sur le terrain professionnel ${ }^{[5]}$ et un site internet nouvellement ouvert dresse de manière très pratique cet inventaire (www. anfh-transfert-acquis-formation.com). 
Concernant l'individu, son sentiment d'efficacité personnelle (SEP) peut interférer sur le transfert. Le SEP est défini par Bandura comme les «jugements que les personnes font à propos de leurs capacités à organiser et réaliser des ensembles d'actions requises pour atteindre des types de performances attendus » ${ }^{[7]}$. C'est ce sentiment qui permet à l'individu de prendre des initiatives et d'affronter les difficultés ${ }^{[8,9]}$. La valeur de la tâche à accomplir, le niveau de performance à atteindre, la perception des chances de réussir sont autant d'éléments qui peuvent influencer le sentiment d'efficacité personnelle et donc la réalisation du transfert d'acquis de formation en situation.

De même, l'environnement de travail, l'attitude bienveillante du supérieur hiérarchique vis-à-vis de ses collaborateurs et plus particulièrement le dispositif d'intégration du cadre de santé novice dans l'organisation de travail, vont influencer sa capacité de transfert des acquis de formation ${ }^{[5]}$. Pour orchestrer au mieux cette intégration, les cadres novices peuvent êtres encadrés par des tuteurs. Le rôle du tuteur est de permettre le travail réflexif vis-à-vis des situations managériales rencontrées. Le tuteur peut, à partir de sa propre expérience, guider le novice dans la mobilisation de ses ressources et ainsi l'aider à élaborer sa compétence, au travers du transfert d'acquis que lui-même a effectué antérieurement dans des situations similaires ${ }^{[3]}$. De plus, l'échange professionnel autour des savoirs expérientiels permet l'élaboration d'un champ d'exercice commun favorable à la coopération au sein d'une équipe d'encadrement $^{[10]}$. Cette coopération entraîne la mutualisation des compétences. La production des compétences est entendue ici comme la combinaison de composantes culturelle, affective, sociale, cognitive et praxéologique au sein de l'équipe ${ }^{[11]}$.

Au cours de la formation, un autre facteur est en mesure de favoriser fortement le transfert d'acquis : la stratégie d'enseignement ${ }^{[5]}$. C'est à ce facteur que notre étude est consacrée et nous nous sommes plus particulièrement intéressés à la stratégie mise en œuvre dans un institut de formation de cadres de santé (IFCS) parisien.

\section{Description d'une stratégie d'enseignement favorisant le transfert des acquis de formation}

Si l'on considère que transférer des acquis de formation (qui sont de l'ordre du «prescrit») signifie mobiliser en situation de travail (« réelle») des capacités (cognitives, techniques, relationnelles) acquises en formation, la gageure pédagogique devient l'élaboration du dispositif de formation développant ce transfert. Le modèle théorique de la dynamique du transfert décrit par Tardif ${ }^{[2]}$ a été appliqué pour l'élaboration d'une stratégie d'enseignement orientée vers le développement de capacités de management des ressources humaines, au sein d'un IFCS (tableau I). La stratégie d'enseignement proposée est constituée de plusieurs séquences planifiées qui comportent pour chacune d'entre elles des activités permettant de travailler les étapes de contextualisation, décontextualisation et recontextualisation, ainsi que des allers et venues entre ces différentes phases. En effet, il semble difficile en pratique de ne travailler qu'une seule étape par séquence, d'autant plus que certains participants à la formation sont déjà en fonction ou ont vécu des situations d'encadrement. Dans la mesure où cette stratégie d'enseignement s'appuie sur un modèle théorique, il a été envisagé que chaque séquence puisse se rapporter un peu plus à un élément constitutif du processus constituant le modèle (par exemple, la première séquence pourrait se rapporter plus spécifiquement à l'étape d'encodage). C'est par le passage d'un processus à un autre et le retour à une étape antérieure en fonction du résultat de chacun d'eux que le transfert s'initie. Par ailleurs, la stratégie proposée respecte un caractère intégrateur ${ }^{[12]}$ en centrant les activités de formation sur des objectifs d'intégration. Cela nécessite de travailler à partir de situations nombreuses et variées qui permettent aux stagiaires cadres d'intégrer plus des méthodes que des concepts. C'est donc au travers d'un curriculum intégratif que le développement des compétences professionnelles se réalise puisque la mobilisation d'apprentissages antérieurs intégrés et non 
Tableau I. Description de la stratégie d'enseignement en alternance, mise en œuvre pour favoriser des apprentissages transférables, en cohérence avec le modèle théorique de la dynamique du transfert selon Tardif ${ }^{[2]}$.

\begin{tabular}{l} 
Ensemble des séquences composant la stratégie \\
d'enseignement en alternance \\
\hline Lieu : centre de formation \\
Enseignements (conférences, travaux dirigés, préparation au \\
stage hospitalier 1, outils d'observation...) \\
Stage hospitalier 1 : 2 à 3 semaines \\
Objectif : observation de la fonction cadre à partir grille d'ob- \\
servation \\
Encadrement par tuteur (cadre de santé) \\
Lieu : centre de formation \\
Exploitation du stage en groupe pluri-professionnel de sta- \\
giaires : analyses de situations (avec formateur) \\
Travail dirigé (avec formateur) : analyse des observations (à \\
partir des grilles) \\
Enseignements (conférences, travaux dirigés, préparation au \\
stage)
\end{tabular}

Stage hospitalier $2: 3$ à 4 semaines

Exemple d'objectifs : repérer une situation managériale récurrente

Encadrement par tuteur (cadre de santé)

Évaluation d'activités professionnelles (épreuves formatives et normatives)

\section{Lieu : centre de formation}

Exploitation du stage en groupe pluri-professionnel de stagiaires : analyses de situations (avec formateur)

Analyse individuelle de la situation managériale repérée donnant lieu à un document écrit

Évaluation du document écrit par un formateur + professionnel en situation de management (épreuve normative)

Argumentation orale par le stagiaire devant formateur + professionnel qui ont évalué préalablement le document écrit (épreuve normative)

Enseignements (conférences, travaux dirigés, préparation au stage)

Stage hospitalier d'approfondissement : 4 semaines Objectif : acquisition de capacités complémentaires Encadrement par tuteur (cadre de santé)

\section{Lieu : centre de formation}

Exploitation du stage d'approfondissement en groupe professionnel de stagiaires : analyses de situations (avec formateur et tuteurs professionnels)

Enseignements (conférences, travaux dirigés) orientés vers la prise de fonction

\begin{tabular}{|l|}
\hline $\begin{array}{l}\text { Élément du modèle théorique de la dynamique } \\
\text { du transfert }\end{array}$ \\
\hline Encodage \\
\hline Céal
\end{tabular}

Certains apprenants ayant déjà fait fonction, ils peuvent envisager la transférabilité de ces enseignements dans leur pratique future (et donc sa représentation)

\section{Représentation de la tâche cible \\ Création d'un modèle mental par l'apprenant}

\section{Accessibilité}

Inventaire par les apprenants d'outils cognitifs participants à la résolution de problèmes. Les exploitations de stage doivent permettre au stagiaire de faire du lien entre la situation de stage et les éléments de connaissances antérieurement acquis

\section{Mise en correspondance}

Les apprenants amorcent liens entre situations-cibles et situations-sources

\section{Adaptation}

Les apprenants déterminent les différences entre situations-cibles et situations-sources

\section{Évaluation}

Permet aux apprenants de fixer les probabilités de réponses aux situations

Les apprenants renforcent la construction de modèles

\section{Génération d'un nouvel apprentissage}

Situation managériales résolues par les cadres novices: transfert d'acquis de formation $=>$ construction de compétences? 
juxtaposés permet en situation l'exercice de la compétence $^{[12]}$.

Cette stratégie sur le «management des ressources humaines » est constituée d'enseignements théoriques sous forme de conférences (les bases du management, les fonctions managériales selon le guide de la Haute autorité de santé ${ }^{[13]}$, - HAS la gestion des compétences...), de travaux dirigés permettant la mobilisation et l'appropriation de méthodes au travers de différentes activités pédagogiques (exemple : jeux de rôles). La place du stage dans cette élaboration est prépondérante puisqu'elle concrétise le principe de l'alternance inscrit dans le projet pédagogique de l'IFCS. Les périodes de stage hospitalier, intercalées par des retours au centre de formation, ont pour objectif de permettre la mobilisation et l'appropriation en situation d'exercice professionnel des capacités acquises au cours de la formation. Les temps de retour à l'IFCS permettent (lors de séances d'exploitation de stage) d'analyser rétrospectivement les situations vécues en stage (tableau I). Les tuteurs de stage sont des cadres de santé expérimentés. Des activités professionnelles sont réalisées durant ces périodes de stage. Les activités pédagogiques, en amont et en aval des périodes de stage, ont pour but de faire mobiliser par les apprenants leurs acquis et d'ancrer des connaissances dans une pratique. L'implication dans la séquence pédagogique de professionnels issus de secteur d'activité hospitalière (tuteur de stage, évaluateur de la situation managériale) et de formateurs de l'IFCS (eux-mêmes cadres de santé) a pour objectif de favoriser l'ancrage des conceptions managériales dans une réalité hospitalière.

La stratégie d'enseignement décrite offre en théorie toutes les caractéristiques permettant le transfert d'acquis de formation. En conséquence, les objectifs de cette étude visent à déterminer : 1) si cette stratégie d'enseignement permet de favoriser le transfert en situation des acquis de formation managériaux chez les cadres de santé novices (c'est-àdire diplômés en décembre 2007) ; 2) si les cadres novices identifient de nouveaux apprentissages lorsqu'ils transfèrent leurs acquis de formation, tout transfert nécessitant des apprentissages d'ajustements à toute situation donnée; 3) quels principaux facteurs individuels et environnementaux ont influencé le transfert des acquis de formation.

\section{Matériels et méthodes}

Population

Les cadres inclus dans l'étude devaient répondre à deux critères : avoir satisfait aux épreuves diplômantes de l'année 2007 (reconnaissance de la fonction par le diplôme) et occuper un poste de cadre de santé en secteur d'activité et non en secteur de formation, depuis six mois. La durée a été déterminée à partir d'une estimation d'un temps jugé suffisant pour avoir un recul nécessaire par rapport au processus du transfert. Cent vingt-deux cadres de santé en exercice ont ainsi été recensés.

\section{Outil de recherche}

Nous nous sommes appuyés sur un outil d'évaluation préalablement élaboré à partir d'une analyse de la littérature ${ }^{[5]}$ dédiée aux conditions de mobilisation de compétence en situation ${ }^{[11,14]}$. Nous avons, pour les besoins de notre étude, complété et adapté cet outil de base. Au final, un questionnaire comprenant 27 questions fermées et cinq questions ouvertes a été élaboré.

Il comprenait trois parties explorant : 1) les caractéristiques des répondants (six questions autour de l'identification et du lieu d'exercice : âge, métier de base, secteur d'activité...); 2) la dynamique de transfert d'acquis en situation (objectifs 1 et 2). Pour cela, il était demandé aux répondants de relater succinctement par écrit une situation managériale qu'ils ont eu à résoudre (et qui se rapportait à une situation d'apprentissage similaire exploitée durant leur formation). Cette description devait comprendre : les personnes impliquées, les étapes suivies et les décisions prises. À partir de cette situation, les répondants devaient identifier les activités pédagogiques utilisées en cours de formation et qui, selon eux, les avaient préparés à résoudre la situation-problème décrite. En identifiant 
eux-mêmes les séquences de formation mobilisées dans leur exercice quotidien, les cadres devaient ensuite cerner les apprentissages d'ajustement mis en œuvre, permettant ainsi d'envisager que le transfert d'acquis de formation était réel (deux questions); 3) les facteurs favorisant le transfert (objectif 3). Des questions ont exploré les facteurs individuels (douze questions dont certaines interdépendantes) et environnementaux (douze questions) influençant le transfert des acquis de formation selon le point de vue des cadres novices.

Les questionnaires ont été envoyés par voie informatique à chacun des 122 cadres de l'étude. Les données recueillies ont été exploitées à l'aide du logiciel Modalisa ${ }^{\circledR}$ pour les questions fermées. Les questions ouvertes ont été traitées manuellement (par catégorisation et occurrences) en fonction de la thématique explorée (situations de travail, analyse post résolution de problème... ).

\section{Resultats}

Caractéristiques des répondants

Après une relance par courriel à tous les cadres novices recensés, 37 questionnaires sur 122 sont revenus, soit un taux de réponse de $30 \%$. Le profil est le suivant : 3 cadres ont moins de 30 ans, 14 se situent entre 30 et 40 ans, 14 entre 40 et 50 ans et 6 ont plus de 50 ans. Presque tous les métiers de base constituant la promotion 2007 sont représentés (exceptés : infirmière de bloc opératoire, ergothérapeute, préparateur en pharmacie). Une grande partie des secteurs d'exercice se retrouvent : pôles d'activités (chirurgical, médical, gérontologique...), crèches, secteurs de support (laboratoire, restauration...).

\section{Analyse des situations managériales relatées par les cadres novices}

Seules 27 situations (sur les 37) ont pu être exploitées; douze relevaient de la "présence au travail », sept de la «gestion de conflits», six de «situations de négociations » et deux de la «gestion de compétences ». Chaque situation a été synthétisée dans un tableau reprenant les éléments concernant : le contexte, les personnes concernées, les étapes de la résolution, les décisions prises par le cadre novice à chacune des étapes. L'exploitation a ensuite permis de repérer : les éléments de la formation utilisés dans la situation, les capacités développées en formation et identifiées comme ayant été mises en œuvre dans la situation (compétences), les fonctions telles que définies par la HAS (tableau II)

La figure 1 présente les activités de la séquence «management» considérées comme utiles par les cadres novices pour résoudre la situation-problème. Seules les activités en lien avec la préparation et l'exploitation des stages sont considérées comme peu ou pas du tout utiles par les cadres novices (respectivement pour 21 et 25 répondants).

Vingt-deux cadres ont analysé la situation à l'issue de la résolution de problème, plus particulièrement avec le collègue cadre et/ou le cadre paramédical de pôle, mais l'équipe d'encadrement au sens large (directeur de soins, cadre supérieur, etc.) a pu être sollicitée. L'analyse de la situation a porté en priorité sur les personnes concernées et l'exposé de la situation. Ce sont ensuite les décisions ainsi que le rôle de chacun dans la situation qui ont été abordés. Enfin, les connaissances et le rôle de l'environnement ont été parfois évoqués. Dix-neuf sur 22 répondants estiment avoir acquis de nouveaux apprentissages (majoritairement en terme de savoir être) à l'issue de ces situations, tels que : "être capable : d'analyser plus rapidement des situations, d'argumenter, de négocier, de mener à bien une discussion, d'effectuer un renforcement positif d'une personne, d'agir avec équité, de ne pas se laisser déborder par ses affects, de ne pas avoir peur de changer les choses, de faire confiance, d'être réactif, d'échanger avec les autres collègues cadres, d'écouter les différents acteurs concernés par la situation... ».

\section{Facteurs influençant le transfert des acquis} de formation

Quatre-vingt-dix-sept \% des répondants $(n=36)$ déclarent s'être sentis en confiance au moment de 


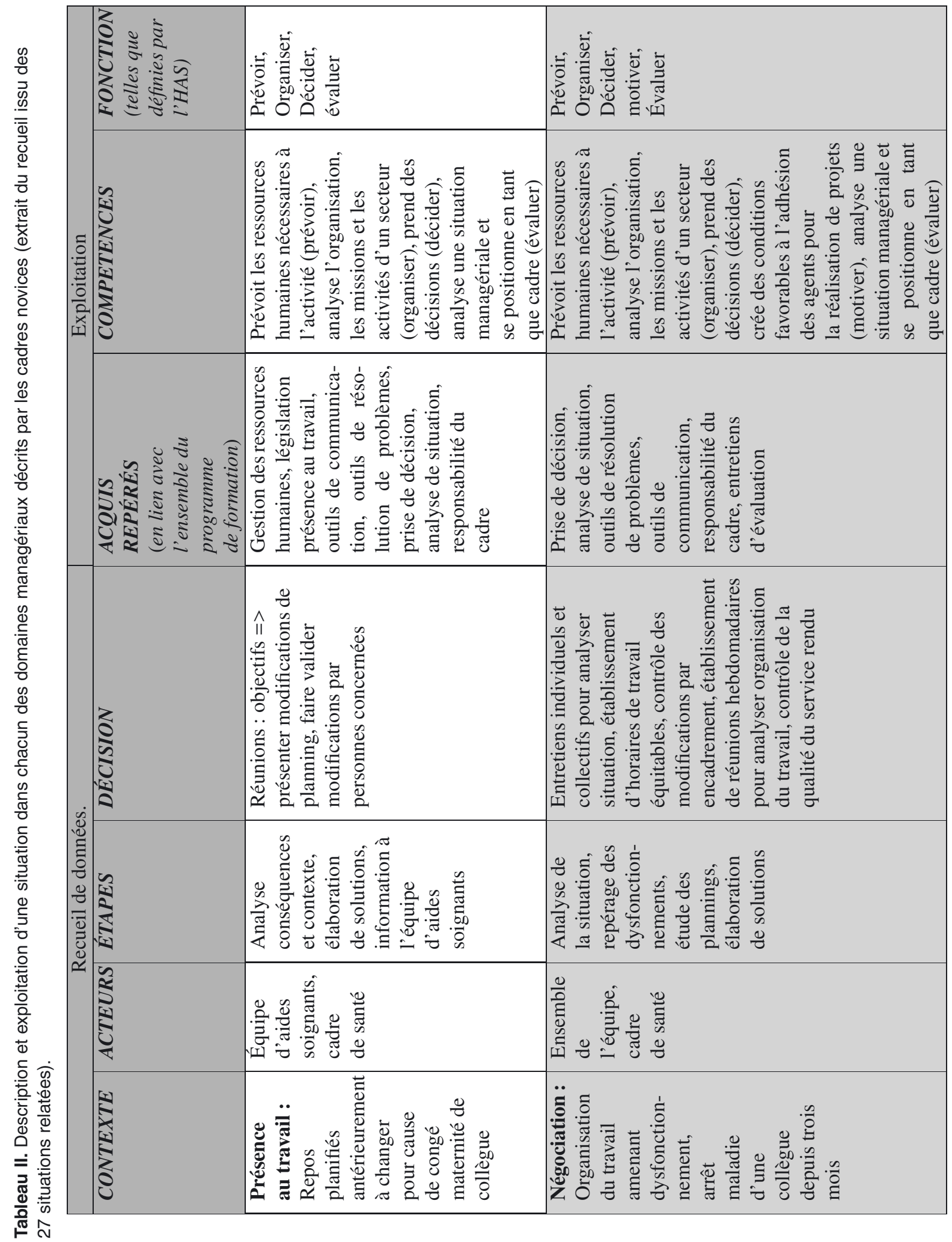




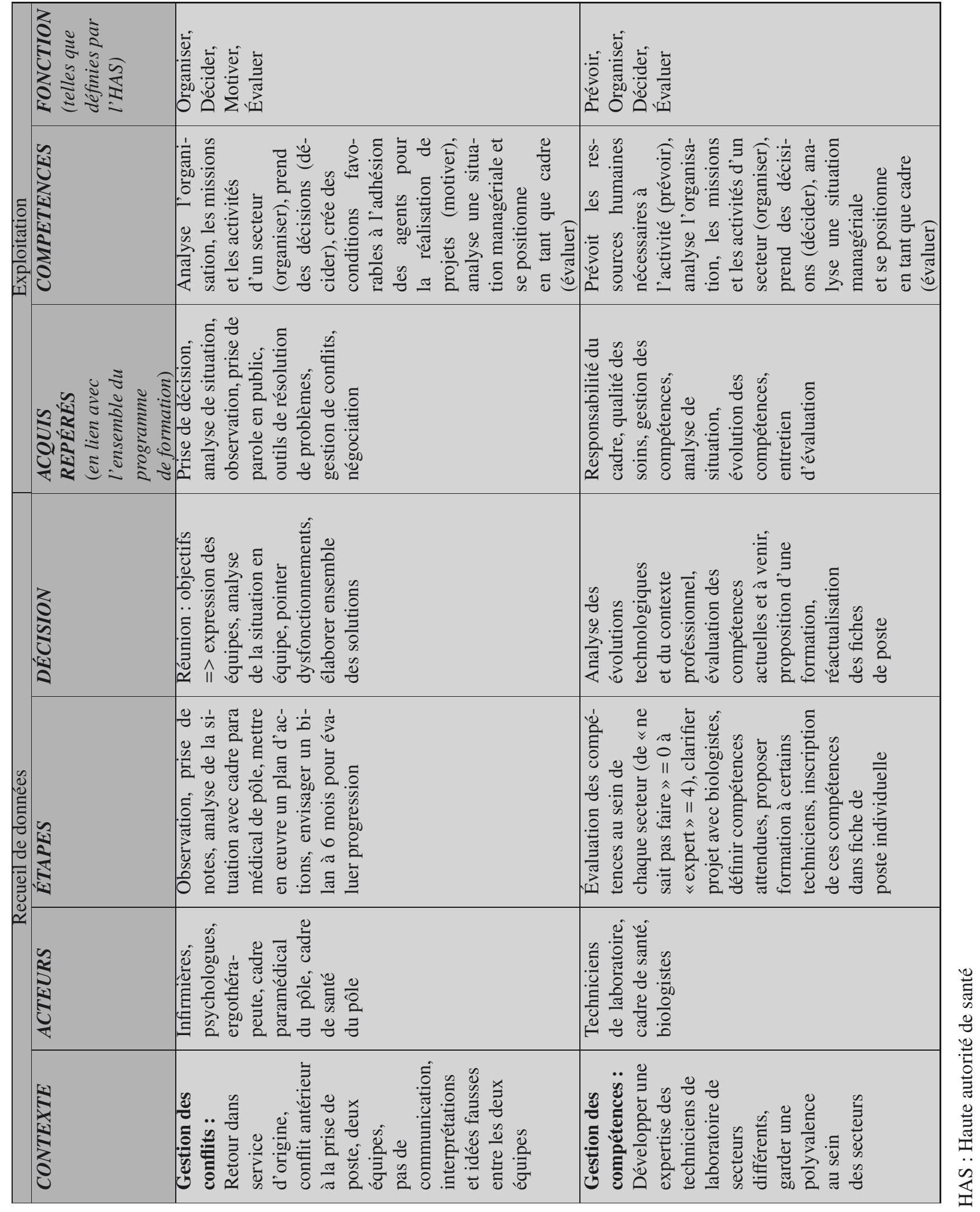


résoudre la situation. Différentes sources à l'origine de cette confiance ont été identifiées (tableau III).

Trente-trois sur 37 répondants estiment ne pas avoir été dépassés au plan émotionnel par cette situation. Pour les autres, l'émotion n'a pas été un frein à la résolution de la situation. Trente-quatre sur 37 cadres novices se sont senti satisfaits à l'issue de la résolution de problèmes (3 peu ou pas du tout). Pour tous, la satisfaction est liée à une issue plutôt positive de la situation.

Les 37 cadres novices s'estiment, à quelques mois de leur prise de poste, compétents dans des domaines managériaux. Cela concerne la gestion des aléas $(n=35)$, la gestion des conflits $(n=34)$, la gestion des ressources humaines $(n=35)$ et le tutorat des étudiants $(n=31)$. Ils se considèrent un peu moins compétents pour la participation aux projets de pôle $(n=7)$, l'évaluation des pratiques professionnelles $(n=8)$, la compétence à motiver une équipe pluridisciplinaire $(n=6)$. Une majorité se ressent compétente pour travailler en équipe d'encadrement $(n=28)$ et se positionner en tant que cadre $(n=34)$.

Parmi les 37 répondants, 24 n'ont pas eu de période d'intégration à leur prise de poste; douze ont bénéficié de cette période d'intégration allant de 15 jours à un mois et ont eu comme tuteur un collègue cadre de santé $(n=10)$ chargé de les accompagner durant cette période.

Cette intégration leur a permis $(n=12)$ : de réaliser une période d'observation systémique, d'avoir des échanges professionnels avec le tuteur et de mieux appréhender leur fonction de cadre de santé.

\section{Discussion}

\section{Limites de l'étude}

Le faible taux de réponse au questionnaire (37 répondants sur 122 envois) est probablement lié à trois raisons :

- la période d'envoi du questionnaire (juin-juillet) qui correspond à une période de congés pour certains.
Tableau III. Classement par catégorie des expressions par les cadres novices des raisons de confiance en soi (illustré par un exemple de verbatim) au moment de la résolution de situation.

\begin{tabular}{|c|c|}
\hline $\begin{array}{c}\text { CATÉGORIES } \\
\text { (par source de confiance en soi) }\end{array}$ & $\begin{array}{c}\text { NOMBRE } \\
\text { d'expression } \\
n=48\end{array}$ \\
\hline $\begin{array}{l}\text { Posture (positionnement, fonction, } \\
\text { grade, être capable de... « « (...) } \\
\text { surtout l'outil principal est le } \\
\text { nouveau regard, } \\
\text { la nouvelle posture» }\end{array}$ & 10 \\
\hline $\begin{array}{l}\text { Expérience professionnelle (savoirs } \\
\text { expérientiels) } \\
\text { "Il s'agissait d'expériences déjà } \\
\text { vécues » }\end{array}$ & 7 \\
\hline $\begin{array}{l}\text { Environnement (climat de travail, } \\
\text { intégration) } \\
\text { «Mes arguments visaient à donner } \\
\text { des conditions de travail accep- } \\
\text { tables» }\end{array}$ & 6 \\
\hline $\begin{array}{l}\text { Renforcement positif du supérieur } \\
\text { (accord de décision) } \\
\text { "Nous avons reçu le soutien } \\
\text { du cadre paramédical de pole» }\end{array}$ & 6 \\
\hline $\begin{array}{l}\text { Formation (résultat de la formation) } \\
\text { «Des solutions ont été proposées et } \\
\text { acceptées grâce aux outils que j’ai } \\
\text { acquis à l'I.F.C.S» }\end{array}$ & 5 \\
\hline $\begin{array}{l}\text { Équipe d'encadrement } \\
\text { (appartenance à un groupe) «Nous } \\
\text { étions deux cadres (...) en accord sur } \\
\text { la démarche à adopter» }\end{array}$ & 3 \\
\hline $\begin{array}{l}\text { Connaissances (réglementation) } \\
\text { «(...) application d'une réglementa- } \\
\text { tion régie par des textes» }\end{array}$ & 3 \\
\hline $\begin{array}{l}\text { Bien fondé de la décision (argumen- } \\
\text { taire développé) } \\
\text { "J'avais pleinement conscience } d u \\
\text { bien fondé de ma demande» }\end{array}$ & 4 \\
\hline $\begin{array}{l}\text { Divers (réactions des interlocuteurs } \\
\text { non connues, questionnement } \\
\text { éthique...) } \\
\text { «J'avais confiance dans les capacités } \\
\text { de réflexions de mon équipe» }\end{array}$ & 4 \\
\hline
\end{tabular}




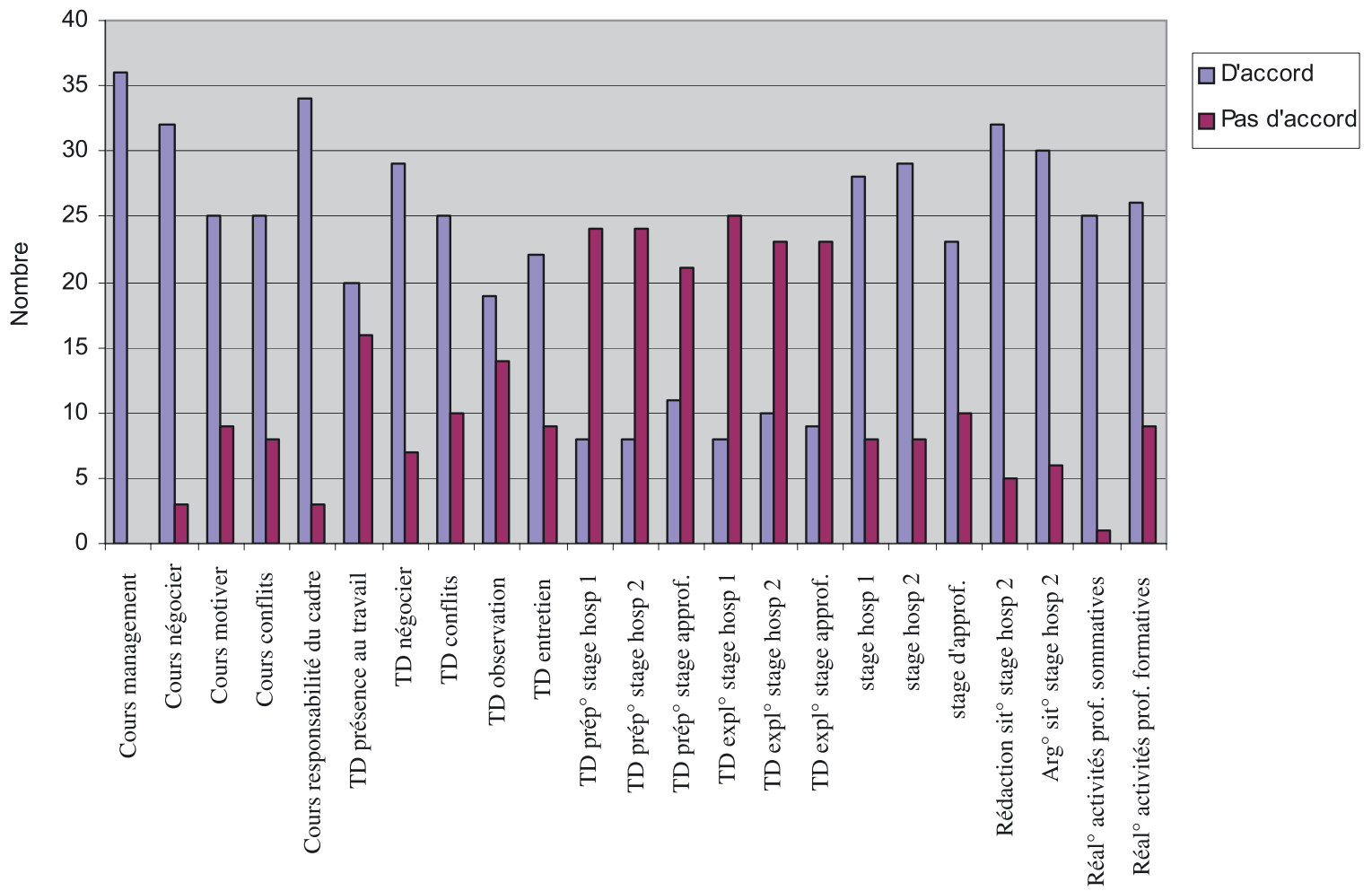

Fig. 1. Éléments de la stratégie d'enseignement considérés par les cadres novices comme utiles pour résoudre les situations décrites (pour une meilleure lisibilité, l'ensemble des réponses « tout à fait d'accord - d'accord » et des réponses « pas d'accord - pas du tout d'accord » ont été respectivement regroupées et les «non réponse » non inclues).

- la modalité de distribution du questionnaire. La voie informatique présente des avantages (moindre coût en papeterie) mais aussi des inconvénients ((in)compatibilité de logiciels entre ordinateurs), ce qui modifiait la structure du questionnaire et ne favorisait pas son renseignement informatique.

- la modalité de récupération du questionnaire (voie courriel). Il était demandé de renseigner le questionnaire informatiquement et de le renvoyer par courriel. Le fichier du questionnaire était long à l'ouverture et peu ergonomique.

Par ailleurs, l'accès aux données académiques des participants n'ayant pas été réalisable dans cette étude, il n'a pas été possible d'analyser et comparer les profils des répondants et des non répondants. Enfin, bien que le questionnaire utilisé dans cette étude s'appuie sur un outil lui-même élaboré à partir d'une revue de la littérature, nous devons admettre que la validité de construit du questionnaire administré ne peut être considéré comme établie.

\section{Discussion des résultats}

\section{Repérage à partir de l'expression des cadres novices, des acquis de formation managériaux transférés}

Pour Frenay ${ }^{[4]}$ le transfert d'acquis ne peut se faire que s'il existe une «structure d'accueil mobilisée au moment de la confrontation », c'est-à-dire une disposition dynamique à confronter la situation rencontrée à ce qui a été enregistré dans une situation antérieure similaire. Dans le dispositif de formation étudié, la situation managériale traitée par le stagiaire cadre à l'issue du stage hospitalier 2, peut être considérée comme la situation initiale qui a favorisé la construction des repères, permettant de disposer des ressources pour résoudre les situations 
auxquelles les cadres novices sont confrontés. C'est ce que semble confirmer le degré d'accord relatif à la perception d'utilité par les cadres novices, de l'activité « rédaction et argumentation de la situation stage hospitalier 2 » pour résoudre la situation décrite (figure 1). L'analyse des situations traitées par les cadres novices semble montrer qu'ils ont recontextualisé la situation cible (situation décrite dans le questionnaire) à partir de références de situations sources (vécues lors de la formation : stages hospitaliers, travaux dirigés...).

Les situations managériales décrites par les répondants (tableau II) font référence à des apprentissages dans les domaines du savoir (législation, présence au travail, responsabilité du cadre... ) mais aussi à du « savoir faire » (réorganisation du travail, analyse de l'activité...) et des savoirs être (écoute, adaptabilité...), que l'on retrouve abordés dans le programme de formation de cet IFCS parisien dont les cadres novices de l'étude sont issus. Même si pour chacun des domaines (présence au travail, gestion des conflits...) les situations décrites par les cadres novices sont différentes, un ensemble de ressources combinées peut se retrouver. La mobilisation en situation de travail de capacités managériales développées en formation, transformées en compétences ${ }^{[11]}$ dans la situation, permet de supposer que des acquis de formation sont transférés par les cadres novices six mois après la prise de poste.

Au regard des situations exposées par les cadres novices, il semble qu'il y a eu "décontextualisation - métacognitivement guidée - d'une stratégie ou d'une procédure » et possible transfert « haut de gamme » des acquis de formation, selon l'expression de Salomon et Perkins ${ }^{[4]}$. En effet, les situations décrites sont analysées en fonction du contexte particulier dans lequel elles se déroulent. Les décisions sont argumentées à partir de références (cognitives, techniques, relationnelles). Le positionnement dans la prise de décision est assumé. La concrétisation de ce transfert «haut de gamme» s'exprime par la production de compétences à résoudre ces situations. Ceci se traduit par la mobilisation des connaissances acquises en formation (composante cognitive) dans un cadre de travail réglementé (composante culturelle) aboutissant à la résolution de la situation (composante praxéologique), la reconnaissance de l'équipe (composante sociale) et à une motivation certaine du cadre novice (composante affective) ${ }^{[11]}$.

Les compétences produites s'appliquent dans l'étude à des situations managériales. À partir des 27 situations managériales exposées, peut-on démontrer la pertinence de la stratégie d'enseignement dont ont bénéficié ces cadres? À l'analyse, l'ensemble du processus de la dynamique du transfert selon Tardif ${ }^{[2]}$ se retrouve (tableau I) : encodage (lors des temps théoriques de formation), représentation de la tâche cible (par analyse de situation sources durant la formation), accessibilité (mobilisation d'outils cognitifs pour résoudre la situation cible), mise en correspondance (liens entre situation source-formation et cible-décrite), adaptation (différence de résolution entre situation cible et situation source), évaluation (résolution de la situation décrite), génération d'un nouvel apprentissage (19 sur 22 identifient de nouveaux apprentissages).

Ceci tendrait à prouver que la stratégie d'enseignement mise en œuvre dans la séquence «management des ressources humaines » de l'IFCS, permettrait de développer la capacité à transférer des acquis en situation. Cependant, une partie des résultats de l'expression des cadres novices à la question concernant les acquis de formation considérés comme utiles interroge directement la stratégie d'enseignement.

Cela concerne les activités pédagogiques en lien avec le dispositif d'alternance (préparation et exploitation de stage). L'alternance dans cette ingénierie a pour ambition d'être intégrative. Ces activités pédagogiques sont construites pour favoriser chez les formés une progression dans l'analyse de situations mais également pour développer leur capacité à transférer. Celle-ci requiert que les formés aient pu travailler sur des situations suffisamment nombreuses et variées ${ }^{[12]}$. C'est le but recherché au travers de ces activités de préparation et d'exploitation de stages. Les réponses des cadres novices (figure 1) montrent que l'aspect intégratif 
recherché par ces activités pédagogiques n'est pas atteint contrairement à l'intérêt des acquis de stages mobilisés pour résoudre les situations exposées. En effet, l'appréciation concernant la réalisation d'activités professionnelles normatives et/ou formatives en stage tend à confirmer le repérage par les cadres novices d'acquis sur le terrain plus qu'en exploitation de stage lors des activités pédagogiques.

Si la stratégie d'enseignement mise en œuvre dans cet IFCS est relativement adaptée, les acquis issus d'un des axes importants de cette stratégie, basée sur l'alternance (préparation des stages par exemple), ne sont pas repérés par les cadres novices comme utiles pour la résolution de situations managériales. La structure du questionnaire n'a pas permis d'en explorer les raisons. Une hypothèse d'explication pourrait être l'insuffisance, dans chacune des séquences de la stratégie, de la phase de contextualisation. Par exemple, la première séquence met peut être trop l'accent sur la conférence au détriment d'une contextualisation considérée comme primordiale dans le modèle de référence proposé ${ }^{[2]}$.

\section{Repérage à partir de l'expression des cadres novices, des facteurs individuels et environnementaux favorisant le transfert d'acquis de formation}

Nos résultats indiquent que la grande majorité des cadres novices s'estiment satisfaits après résolution de la situation exposée. Or, avoir solutionné la situation (de niveau de complexité variable) renforce certainement la confiance en soi dans la capacité à résoudre les situations à venir ${ }^{[5]}$. Cela est d'autant plus vrai que ces professionnels doivent d'une certaine manière faire leurs preuves. Cette confiance en soi (tableau III) s'appuie principalement sur une perception forte de leur fonction, de leur identité ou de leur positionnement en tant que cadre $(n=10)$, mais aussi sur leurs savoirs d'expérience développés au cours de leur activité professionnelle $(n=7)$. Elle s'appuie également sur des connaissances (cadre législatif donnant une certaine validité à la décision) et des capacités relationnelles et humaines appliquées pertinemment à la situation (formation, bien fondé de la décision).
Par ailleurs, 34 cadres novices (sur les 37 répondants) s'estiment satisfaits après résolution de la situation exposée. Cette réussite dans la résolution de ces situations semble renforcer le sentiment d'efficacité personnelle (SEP) ${ }^{[7]}$ qui est une des variables favorisant le transfert d'acquis ${ }^{[5]}$. Si parfois $(n=3)$ il y a insatisfaction, celle-ci est liée à la perception que les résultats obtenus ne sont pas ceux escomptés dans le temps imparti à la résolution de la situation (des décisions prises trop tardivement par exemple).

Les cadres novices ne semblent pas trop dépassés sur le plan des émotions face à ces situations managériales. Ceux qui l'ont été «en partie» au moment de résoudre la situation le rapprochent de la notion de qualité des soins et de sécurité à garantir par le cadre. Cette notion, prégnante au moment de la résolution du problème, peut engendrer un bref moment d'inquiétude. Celui-ci est rapidement levé par la résolution du problème et le recours éventuel aux collègues.

Un autre élément favorisant le transfert est la relation avec le supérieur hiérarchique se traduisant par un soutien et un renforcement positif. Comme l'explique Gagnayre ${ }^{[5]}$ la relation positive avec le supérieur hiérarchique est une des variables influant sur le transfert d'acquis. Cette relation de renforcement positif transparaît dans les réponses des cadres (tableau III). L'analyse post situation porte en partie sur les décisions prises par les cadres novices. Elle se fait avec l'équipe d'encadrement au sens large. La référence à des cadres experts permet aux cadres novices de conforter leurs acquis, de partager de l'expérience et ainsi de renforcer leurs propres compétences. Ceci se confirme par les domaines dans lesquels les cadres novices s'estiment compétents. La «gestion des aléas » et la «gestion des ressources humaines » sont majoritairement mentionnées, ce qui est en lien avec les situations rencontrées au quotidien par les cadres novices. Ils pointent cependant pour une faible partie (3 sur 37) le caractère débutant de leur fonction puisque, pour ceux-ci, «se positionner en tant que cadre » reste à renforcer.

Enfin, un dernier facteur lié à l'environnement semble faciliter le transfert des acquis ${ }^{[5]}$. Il concerne 
un type d'organisation de travail offrant aux cadres novices une période d'intégration ; 10 cadres sur les 12 qui ont eu une période d'intégration affirment que cela permet de "mobiliser les acquis de formation ». Cependant beaucoup de cadres retrouvent leur établissement d'origine et par conséquent ne sollicitent pas cette période. De plus le manque actuel de personnel au sein de l'équipe d'encadrement pourrait constituer un frein à une telle demande.

\section{Perspectives}

L'étude démontre que la dynamique de transfert est davantage initiée, dans la stratégie d'enseignement étudiée, par les enseignements, les travaux dirigés, les stages, que par la préparation et l'exploitation des stages. Cela doit amener une réflexion pédagogique concernant les objectifs poursuivis lors de ces activités. Cela suppose de transformer des objectifs d'apprentissage ( «L'étudiant sera capable de... ») en objectifs d'intégration ( « Au cours du stage hospitalier 2, l'étudiant analysera avec son tuteur une situation managériale »).

Ceci pourrait renforcer un curriculum intégratif. Par exemple, à partir de cette situation analysée avec le tuteur de stage, l'activité pédagogique en centre de formation serait de faire expliciter au formé comment il a mobilisé ses acquis dans cette situation (métacognition). L'objectif du formateur serait alors d'aider l'apprenant à faire des liens entre les différents apprentissages. D'autres activités pédagogiques autour de la construction (y compris par les formés) de situations-problèmes significatives seraient aussi à envisager. Il est à supposer que ce dispositif puisse permettre de renforcer, chez les stagiaires-cadres, la capacité de transfert déjà initiée par le dispositif de formation actuel.

Les autres éléments favorisants concernent les facteurs individuels et l'environnement. Les facteurs individuels pourraient être sollicités à partir de situations de formation développant ou renforçant le sentiment d'efficacité personnelle. Pour l'organisation du travail et son environnement, cela impliquerait une collaboration avec les secteurs d'activité des cadres novices pour optimiser la dynamique du transfert, en s'appuyant entre autres sur un dispositif d'intégration construit. En effet «il n'y a pas de terme au transfert ${ }^{[1]}$. Cette dynamique doit être entretenue pour que des personnels puissent rester compétents dans un contexte évolutif. L'analyse de situations, initiée par le cadre novice lors de son arrivée, permet de développer des compétences individuelles mais également collectives. Cet échange (cadres novices - cadres expérimentés) lorsqu'il devient une pratique professionnelle régulière, garantit une certaine qualité de production managériale.

\section{Conclusion}

Nous avons analysé l'influence du facteur liée à la stratégie d'enseignement mise en œuvre dans un IFCS parisien, dans la perspective du transfert d'acquis. Les résultats tendraient à confirmer que des acquis de formation sont transférés par les cadres de santé novices issus de ce programme de formation. La stratégie d'enseignement mise en œuvre dans l'IFCS et qui porte sur le «management des ressources humaines » répondrait à la demande concernant la préparation des cadres de proximité aux enjeux managériaux actuels. Les résultats mettent en évidence que les séquences dédiées à la préparation et à l'exploitation des stages sont à revoir au sein du dispositif de formation de l'IFCS. La formation tend à rendre opérationnels les formés : ils résolvent des situations de niveaux de complexité différents en regard d'éléments divers et variés. Ils semblent réaliser un transfert d'acquis de formation qui peut être qualifié de «haut de gamme». Il apparaît incontournable de renforcer dans la stratégie d'enseignement, l'alternance intégrative, enjeu fondamental de la construction des compétences professionnelles collectives.

La question du transfert d'acquis de formation reste cependant posée. Si l'étude démontre une certaine mobilisation cognitive et praxéologique de la formation dispensée, elle ne permet pas d'affirmer la présence d'un transfert d'acquis de formation puisque cela supposerait de s'intéresser aussi à 
l'autre versant du transfert : les dynamiques de travail en contexte professionnel avéré. Dans la mesure où les facteurs liés à l'organisation du travail n'ont pas été pleinement explorés, l'affirmation de transfert d'acquis de formation reste partielle. Il serait nécessaire de donner une suite à cette étude pour approfondir les aspects liés à l'organisation du travail. Cependant, le fait qu'une ingénierie de formation de cadres de proximité interpelle l'organisation du travail par le problème de l'apprentissage laisse envisager de possibles aménagements conduisant, à terme, à ce que l'on nomme dans le milieu du travail : les organisations apprenantes. Il s'agit alors, par la mise en œuvre de conditions spécifiques, de concevoir l'activité professionnelle comme une possibilité d'apprendre pour les acteurs. Ce dispositif permettrait ainsi de dépasser les limites que rencontrent souvent les formations initiales.

\section{Contributions}

Sylvie Kinet a mené l'étude dans le cadre d'un mémoire de master professionnel «Éducationclinique et santé; spécialité : ingénierie des formations en santé ». Elle a élaboré le protocole de l'étude, collecté et analysé les données, interprété les résultats et rédigé les versions initiale et finale du manuscrit; Claire Marchand a dirigé l'étude et participé à la rédaction des versions initiale et finale du manuscrit. Jeanne Rallier et Rémi Gagnayre ont participé à la révision critique et à l'approbation de la version finale.

\section{Références}

1. Develay M. De l'impossible et de la nécessaire pensée du transfert. Education 1998;15:8-10.

2. Tardif J. Le transfert des apprentissages. Montréal : Éditions Logiques, 1999.

3. Sliwka C, Perez P. La transférabilité des compétences, in Les cadres de santé, des cadres de métier. Rueil Malmaison : Édition Lamarre, Collection Gestion des Ressources Humaines : fonction cadre de Santé, 2007:1-9

4. Frenay M. Le transfert d'apprentissage, L'adulte en formation, regards pluriels. Bruxelles, Paris : Ed. De Boeck et Larcier SA, 1996:37-56

5. Gagnayre R. Les facteurs qui influencent le transfert : une revue de littérature. collaboration Format-Santé-ANFH Picardie (2006-2008). 2007. [On-line] Disponible sur : http://www . transfert-acquis-formation.com/ page/biblio.php

6. Legendre R. Dictionnaire actuel de l'éducation, Montréal, Édition Guérin, $3^{\mathrm{e}}$ éd, 2005.

7. François $\mathrm{Ph}$. Sentiment d'efficacité et compétences : une approche sociale cognitive. Éducation permanente 1998;135:45-55.

8. Bandura A. «J'y arriverai » : le sentiment d'efficacité personnelle. Sciences Humaines 2004;148:42-5.

9. Pelaccia T, Delplancq H, Triby E, Leman C, Bartier JC, Dupeyron JP. La motivation en formation : une dimension réhabilitée dans un environnement d'apprentissage en mutation. Pédagogie Médicale 2008;9:103-21.

10. Chague V. Comment améliorer la coopération au sein de l'équipe soignante ? Soins cadres de santé 2008 (sup. 65):8-12.

11. Wittorski R. De la fabrication des compétences. Éducation permanente 1998;35:57-69.

12. Roegiers $X$ (avec la collaboration de De Ketele JM). Une pédagogie de l'intégration. Compétences et intégration des acquis dans l'enseignement. Bruxelles, Ed. De Boeck, $2^{\mathrm{e}}$ ed. 2001.

13. Haute Autorité de Santé (HAS) - Guide pour l'autodiagnostic des pratiques de management en établissement de santé. Direction de l'accréditation et de l'évaluation des pratiques professionnelles. 2005 [On-line] Disponible sur : www .has-sante.fr

14. Crozet C, Marchand C, Iguenane J, Gagnayre R. Recueil d'outils d'évaluation facilitant l'apprentissage, Bobigny, Ed. DPSS-U.F.R SMBH, Université Paris 13, 2005 [On-line] Disponible sur : http://www . transfert-acquis-formation . com/upload/file/autrespdf/ pdf-questionnaire-54-58-59-41.pdf

Correspondance et offprints : Sylvie Kinet, Institut de formation des cadres de santé de l'Assistance Publique-Hôpitaux de Paris, France.

Mailto : sylvie.kinet2@sante.gouv.fr 